\title{
Community-acquired methicillin-resistant Staphylococcus auereus in a Malaysian tertiary centre in year 2009
}

\author{
Z Zainol Rashid ${ }^{1 *}$, N Bahari', A Othman ${ }^{1}$, R Jaafar ${ }^{1}$, NA Mohamed $^{1}$, I Jabbari ${ }^{1}$, A Sulong ${ }^{1}$, R Hashim², N Ahmad ${ }^{2}$ \\ From International Conference on Prevention \& Infection Control (ICPIC 2011) \\ Geneva, Switzerland. 29 June - 2 July 2011
}

\section{Introduction / objectives}

Community-Acquired Methicillin-Resistant Staphylococcus aureus (CA-MRSA) is a pathogen recognized to be distinct from hospital-acquired MRSA phenotypically and genotypically. We aimed to identify CA-MRSA cases in UKMMC, their antibiotic susceptibility patterns and genotypic characteristics.

\section{Methods}

Cases were identified prospectively from January to December 2009, where culture and antibiotic susceptibility results yielding pauciresistant MRSA isolates were suspected as CA-MRSA. The patients' clinical data were collected and their specimens were sent for molecular confirmation and analysis at IMR.

\section{Results}

Five cases of CA-MRSA were identified. The isolates had multisensitive pattern on antibiotic susceptibility testing and were resistant to only penicillin and oxacillin. All cases were skin and soft-tissue infections, namely diabetic foot with gangrene, infected scalp haematoma, philtrum abscess in a healthcare worker, thrombophlebitis complicated with abscess and infected bedsore. All five cases were confirmed MRSA by detection of mecA gene. SCCmec typing (ccr gene and mec complex gene) revealed SCCmec type IV for all except the infected bedsore case. Panton-Valentine Leucocidin gene was positive in all isolates.

\section{Conclusion}

The clinical confines between methicillin-sensitive Staphylococcus aureus, CA-MRSA and "nosocomial CAMRSA" are indistinct. Early recognition is necessary in instituting appropriate antibiotics and infection control measures. Continued surveillance of pauciresistant MRSA and molecular analysis is useful to identify emerging strains and their epidemiology and transmission, both in the community as well as healthcare settings.

\section{Disclosure of interest}

None declared.

\section{Author details}

'Dept of Medical Microbiology \& Immunology, Faculty of Medicine,Universiti Kebangsaan, Malaysia. ${ }^{2}$ Bacteriology Unit, Institute of Medical Research, Kuala Lumpur, Malaysia.

Published: 29 June 2011

doi:10.1186/1753-6561-5-S6-P18

Cite this article as: Zainol Rashid et al: Community-acquired methicillinresistant Staphylococcus auereus in a Malaysian tertiary centre in year 2009. BMC Proceedings 2011 5(Suppl 6):P18. 